\title{
Maintenance of C. elegans ${ }^{*}$
}

\author{
Theresa Stiernagle ${ }^{\S}$, Caenorhabditis Genetics Center, University of \\ Minnesota, Minneapolis, MN 55455 USA
}

\section{Table of Contents}

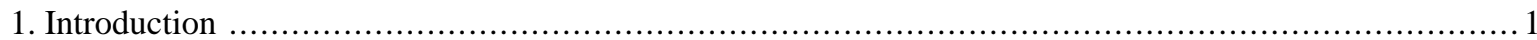

2. Acquiring strains from the Caenorhabditis Genetics Center (CGC) ....................................... 1

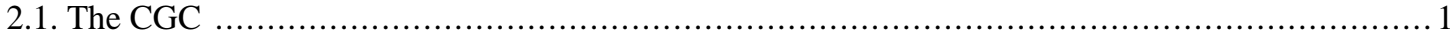

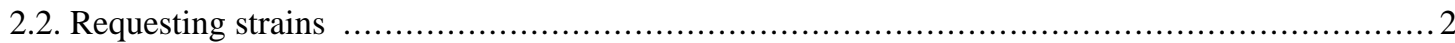

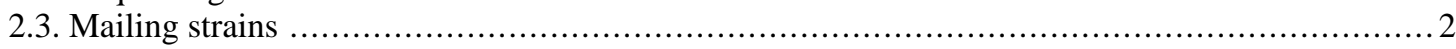

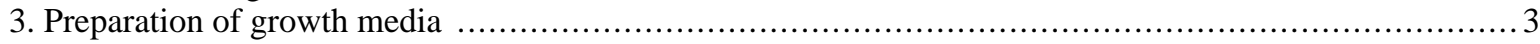

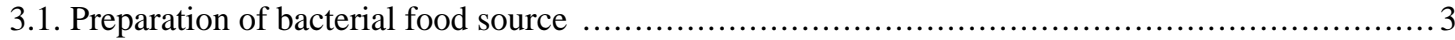

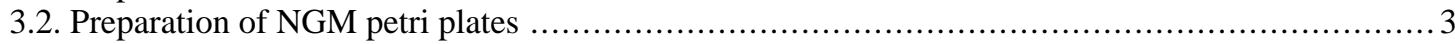

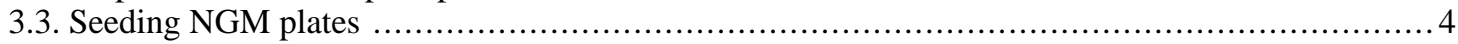

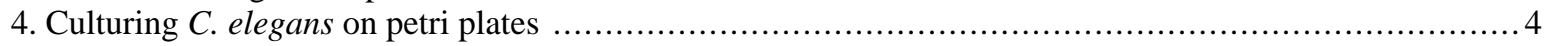

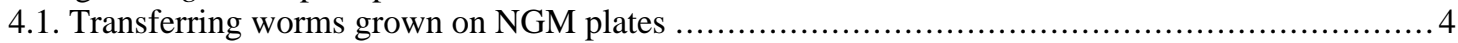

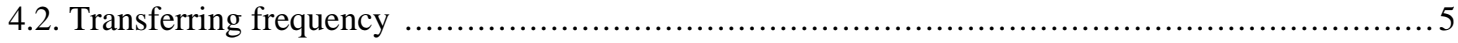

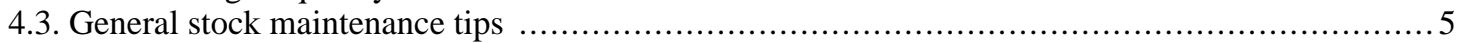

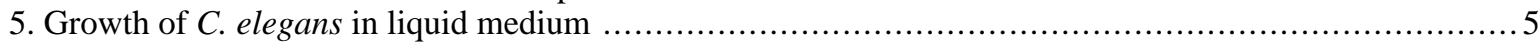

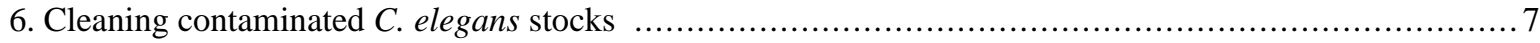

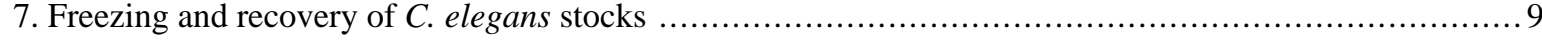

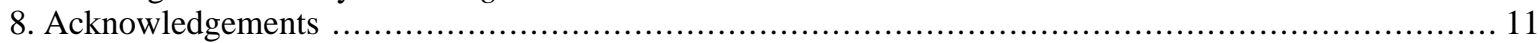

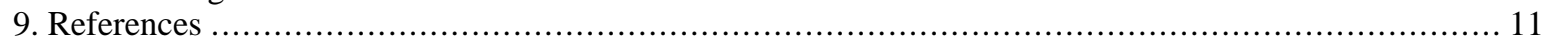

\section{Introduction}

This chapter discusses the maintenance of $C$. elegans in the laboratory. Topics include the acquisition of worm strains from the Caenorhabditis Genetics Center (CGC), methods of culture and transfer, decontamination of stocks, synchronizing and staging cultures, and procedures for freezing worms for long-term storage and for recovering them from the frozen state.

\section{Acquiring strains from the Caenorhabditis Genetics Center (CGC)}

\subsection{The CGC}

The CGC was established at the University of Missouri, Columbia, in 1979. In 1992 the CGC moved from Missouri to the University of Minnesota, St. Paul. Funded through a contract with the National Institutes of Health

\footnotetext{
*Edited by David Fay. WormMethods editor, Victor Ambros. Last revised May 7, 2005. Published February 11, 2006. This chapter should be cited as: Stiernagle, T. Maintenance of C. elegans (February 11, 2006), WormBook, ed. The C. elegans Research Community, WormBook, doi/10.1895/wormbook.1.101.1, http://www.wormbook.org.

Reprinted from pp. 51-67, Maintenance of C. elegans, by T. Steiernagle in C. elegans: A Practical Approach, edited by Hope, Ian (1999), by permission of Oxford University Press.

${ }^{\S}$ To whom correspondence should be addressed. E-mail: cgc@umn.edu
} 
National Center for Research Resources (NIH NCRR), the basic mission of the CGC is to provide C. elegans strains and information to scientists initiating or continuing research using C. elegans. The CGC strives to acquire and have available for distribution stocks representing at least one mutant allele of each published gene and all chromosome rearrangements (deficiencies, duplications, translocations, inversions). In addition, many wild-type isolates of $C$. elegans and several species closely related to C. elegans are available. The CGC maintains an up-to-date bibliographic list of all articles, reviews and books that discuss C. elegans. The Worm Breeder's Gazette (WBG), a newsletter that is published three times per year, is available in hard copy from the CGC for a small two-year subscription fee, and it is available free of charge on the internet. The WBG contains a C. elegans bibliographic update, announcements and news, a directory of $C$. elegans researchers, and abstracts submitted by $C$. elegans researchers who briefly describe their current work.

The jobs of maintaining the C. elegans genetic nomenclature and the genetic map are handled by the CGC as a subcontract under the directorship of Jonathan Hodgkin at the MRC Laboratory of Molecular Biology, Cambridge, England. A second subcontract helps Leon Avery (Southwestern Medical Center, University of Texas, Dallas) maintain the C. elegans World Wide Web WWW Server (http://elegans.swmed.edu/) as a source of information about C. elegans, including the services of the CGC and descriptions of strains that are available.

\subsection{Requesting strains}

Wild-type and over 3,000 mutant strains of $C$. elegans are available at no cost to researchers at educational institutions or non-profit organizations. To obtain strains, submit a written request (letter, fax, or email) describing the strains, genes, or alleles desired and a brief statement of the intended use of the worms. Include within the request the name, complete mailing address and phone numbers of the person requesting the strain(s).

Researchers at commercial or for-profit organizations are required to pay a fee of $\$ 100.00$ plus postage for each strain of worms or bacteria ordered. A "Commercial Sector Order Form" is available from the CGC; a completed form must be received by the CGC before strains will be sent to commercial organizations.

All requests for strains or information should be sent to: Caenorhabditis Genetics Center, University of Minnesota, 250 Biological Sciences Center, 1445 Gortner Avenue, St. Paul, MN 55108-1095 USA. Requests can also be faxed [(612) 625-5754] or emailed [cgc@umn.edu].

\subsection{Mailing strains}

Upon receipt of a strain request, the CGC will send a response confirming that the order has been received. The requested strains are either thawed from a freezer vial or recovered from a starved plate kept in an incubator at $11^{\circ} \mathrm{C}$. Animals of each strain are placed on freshly prepared Nematode Growth Medium (NGM) petri plates that have been spread with $E$. coli as a source of food and allowed to reproduce for several days. The visible phenotype of the stock is noted to confirm that it matches the published description. The petri plates are then wrapped with Parafilm and mailed to the requester. It takes 7-10 days for a strain request to be received, processed and filled. If a requested strain has a temperature sensitive lethal phenotype, the stock is allowed to starve at permissive temperature before the plates are shipped, a step that takes an additional 3-4 days. This results in the formation of heat-resistant dauer larvae (see Genetic mapping and manipulation-Introduction and basics).

Strains are sent on NGM petri plates with the worms still feeding on E. coli OP50 (or starved, as mentioned above). The plates are shipped in bubble-lined mailing bags via First Class or Air Mail. Postage costs are paid by the CGC except for commercial orders. Strains can be shipped via Federal Express if the requester provides the CGC with a Federal Express account number to bill the courier costs. Strains sent outside the USA have a United States Postal Service Customs (CN22) sticker applied to the package. The sticker declares that the package contains "non-pathogenic, non-parasitic biological specimens of no commercial value." Occasionally the customs departments of other countries require that additional information be applied to the package. In such cases, the requester should supply the CGC with the needed information.

Strains are sent with a Strain Information Sheet, which gives the genotype, phenotype, culturing conditions and derivation of the stock, as well as a bibliographic reference. Requesters are asked to inform the CGC of the date the strains were received and their condition on arrival. The CGC should be acknowledged in any publication that results from the use of strains acquired from the CGC. 


\section{Preparation of growth media}

\subsection{Preparation of bacterial food source}

Although C. elegans can be maintained axenically (Avery, 1993), it is difficult, and the animals grow very slowly. C. elegans is usually grown monoxenically in the laboratory using E. coli strain OP50 as a food source (Brenner, 1974). E. coli OP50 is a uracil auxotroph whose growth is limited on NGM plates. A limited bacterial lawn is desirable because it allows for easier observation and better mating of the worms. A starter culture of $E$. coli OP50 can be obtained from the CGC or can be recovered from worm plates. Use the starter culture to isolate single colonies on a streak plate of a rich medium such as LB agar [10 g Bacto-tryptone, $5 \mathrm{~g}$ Bacto-yeast, $5 \mathrm{~g} \mathrm{NaCL}, 15 \mathrm{~g}$ agar, $\mathrm{H}_{2} \mathrm{O}$ to 1 litre, $\mathrm{pH}$ 7.5] (Byerly et al., 1976). Using a single colony from the streak plate, aseptically inoculate a rich broth, such as L Broth $\left[10 \mathrm{~g}\right.$ Bacto-tryptone, $5 \mathrm{~g}$ Bacto-yeast, $5 \mathrm{~g} \mathrm{NaCl}, \mathrm{H}_{2} \mathrm{O}$ to 1 litre, $\mathrm{pH}$ to $7.0 \mathrm{using} 1 \mathrm{M}$ $\mathrm{NaOH}$. Put $100 \mathrm{ml}$ into $250 \mathrm{ml}$ screw-cap bottles and autoclave. The bottles of media can be stored at room temperature for several months (Byerly et al., 1976)]. Allow inoculated cultures to grow overnight at $37^{\circ} \mathrm{C}$. The $E$. coli OP50 solution is then ready for use in seeding NGM plates. The E. coli OP50 streak plate and liquid culture should be stored at $4^{\circ} \mathrm{C}$ and will remain usable for several months.

\subsection{Preparation of NGM petri plates}

C. elegans is maintained in the laboratory on Nematode Growth Medium (NGM) agar which has been aseptically poured into petri plates (Brenner, 1974). Several sizes of petri plates are available and can be purchased from companies such as Nunc or Falcon. Smaller plates (35 mm diameter) are useful for matings or when using expensive drugs. Medium size plates (60 mm diameter) are useful for general strain maintenance, and larger plates (100 mm diameter) are useful for growing larger quantities of worms, such as for certain mutant screens. The NGM agar medium can be poured into petri plates easily and aseptically using a peristaltic pump. The CGC uses a Wheaton Unispense liquid dispenser (Wheaton Science Products). This pump can be adjusted so that a constant amount of NGM agar is dispensed into each petri plate. A constant amount of agar in the plates reduces the need for refocusing the microscope when you switch from one plate to another. When desired, drugs (e.g., levamisole, streptomycin or nystatin) can be added to the NGM solution just prior to its being poured Caldicott et al., 1994).

Protocol 1. Preparation of NGM plates

Equipment and Reagents

- $\mathrm{NaCl}$

- Agar

- $\quad$ Peptone

- $\quad 5 \mathrm{mg} / \mathrm{ml}$ cholesterol in ethanol (Do not autoclave!)

- $\quad 1 \mathrm{M} \mathrm{KPO}_{4}$ buffer pH $6.0\left(108.3 \mathrm{~g} \mathrm{KH}_{2} \mathrm{PO}_{4}, 35.6 \mathrm{~g} \mathrm{~K}_{2} \mathrm{HPO}_{4}, \mathrm{H}_{2} \mathrm{O}\right.$ to 1 litre $)$

- $\quad 1 \mathrm{M} \mathrm{MgSO}_{4}$

- $\quad$ Petri plates

- $\quad$ Peristaltic pump

Methods

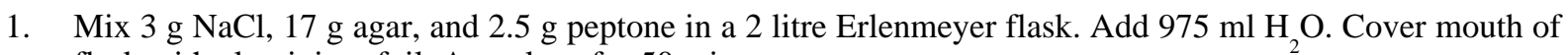
flask with aluminium foil. Autoclave for $50 \mathrm{~min}$.

2. Cool flask in $55^{\circ} \mathrm{C}$ water bath for $15 \mathrm{~min}$.

3. Add $1 \mathrm{ml} 1 \mathrm{M} \mathrm{CaCl}_{2}, 1 \mathrm{ml} 5 \mathrm{mg} / \mathrm{ml}$ cholesterol in ethanol, $1 \mathrm{ml} 1 \mathrm{M} \mathrm{MgSO}_{4}$ and $25 \mathrm{ml} 1 \mathrm{M} \mathrm{KPO}_{4}$ buffer. Swirl to mix well. 
4. Using sterile procedures, dispense the NGM solution into petri plates using a peristaltic pump. Fill plates 2/3 full of agar.

5. Leave plates at room temperature for 2-3 days before use to allow for detection of contaminants, and to allow excess moisture to evaporate. Plates stored in an air-tight container at room temperature will be usable for several weeks.

It may be desirable to use 96- or 24-well microtiter plates when using expensive drugs or screening a large number of individual animals. For 24 -well plates, use $1.5 \mathrm{ml} \mathrm{NGM}$ agar and seed with an $E$. coli OP50 lawn (Hartman and Herman, 1982). 96-well plates can be filled with $50 \mu \mathrm{l}$ of a $1 \%$ (w/w) suspension of $E$. coli OP50 in S Medium (see Protocol 2) (Hirsh et al., 1976). 96-well plates can also be filled with $50 \mu$ of NGM liquid with $E$. coli HB101 each well. To do this, packed E. coli HB101 cells are suspended in 4X their volume of NGM liquid, assuming $1 \mathrm{~g}$ of cells equals $1 \mathrm{ml}$. One volume of the $E$. coli suspension is then added to 24 volumes of NGM liquid (Ralph Clover, personal communication). E. coli HB101 is available from the CGC. Care should be taken to prevent worms from crawling between wells; only one strain of C. elegans should be used per microtiter plate so that there is no possibility of strains mixing.

\subsection{Seeding NGM plates}

Using sterile technique, apply approximately $0.05 \mathrm{ml}$ of E. coli OP50 liquid culture to small or medium NGM plates or $0.1 \mathrm{ml}$ to large NGM plates using a pipet. If desired, the drop can be spread using the pipet tip or a glass rod. Spreading will create a larger lawn, which can aid in visualizing the worms. Take care not to spread the lawn all the way to the edges of the plate; keep the lawn in the center. The worms tend to spend most of the time in the bacteria. If the lawn extends to the edges of the plate the worms may crawl up the sides of the plate, dry out and die. Allow the E. coli OP50 lawn to grow overnight at room temperature or at $37^{\circ} \mathrm{C}$ for 8 hours (cool plates to room temperature before adding worms). Seeded plates stored in an air-tight container will remain usable for 2-3 weeks.

\section{Culturing $C$. elegans on petri plates}

\subsection{Transferring worms grown on NGM plates}

C. elegans is transparent and can be visualized using a dissecting stereomicroscope equipped with a transmitted light source. The CGC uses Wild Leitz model M5A or Zeiss model SV6. Standard 10X eyepieces and objectives which range from $0.6 \mathrm{X}$ to $5 \mathrm{X}$ (total magnification of $6 \mathrm{X}$ to $50 \mathrm{X}$ ) are widely used.

Several methods are used for transferring C. elegans from one petri plate to another. A quick and convenient method is "chunking", wherein a sterilized scalpel or spatula is used to move a chunk of agar from an old plate to a fresh plate. There will usually be hundreds of worms in the chunk of agar. The worms will crawl out of the chunk and spread out onto the bacterial lawn of the new plate. This method works well for transferring worms that have burrowed into the agar or are difficult to pick individually (such as on a starved plate). The chunking method is fine for transferring homozygous stocks but it is not advisable if the population is heterozygous or if a stock must be maintained by mating.

Another method that will transfer many worms is to use strips of sterilized filter paper which have been cut to $1 / 2$ to $1 / 4$ inch wide and 2-3 inches long. The sterilized filter paper is gently set upon the petri plate, where it absorbs moisture and picks up worms. The filter paper is then touched to a fresh NGM plate where the worms are deposited. Discard the filter paper after use. This method is also fine for transferring homozygous stocks but it is not advisable if the population is heterozygous or must be maintained by mating.

A third method is to pick single animals with a worm picker. A worm picker can be made by mounting a 1-inch piece of 32 gauge platinum wire into either the tip of a Pasture pipet or in a bacteriological loop holder. Platinum wire heats and cools quickly and can be flamed often (between transfers) to avoid contaminating the worm stocks. The end of the wire, used for picking up worms, can be flattened slightly with a hammer and then filed with an emery cloth to remove sharp edges; sharp points can poke holes in the worms and kill them or make holes in the agar. The tip of the wire can be fashioned to your liking. Some people prefer a flattened end, while others prefer a slight bend that forms a hook. It takes a bit of experience with a worm picker to avoid poking holes in the agar. Worms crawl into the holes, making it difficult to see or pick them. 
To pick a worm identified under the dissecting microscope, slowly lower the tip of the wire and gently swipe the tip at the side of the worm and lift up. Another method is to get a blob of E. coli OP50 on the end of the picker before gently touching it to the top of the chosen worm. The worm will stick to the bacteria. Several animals at a time can be picked by this method, although worms left too long on the pick will desiccate and die. To put a picked worm on a fresh plate, slowly lower the tip of the worm picker, gently touch the surface of the agar, and hold it there to allow the worm to crawl off of the picker.

\subsection{Transferring frequency}

The frequency that you need to transfer your worms depends on their genotype, the temperature at which you grow them, and what you plan to do with them. Heterozygotes and mating stocks should be transferred every 1-3 generations; it is easier to transfer them if you do it before the plates have become starved. If you want to transfer individual animals from a starved plate you may find it easier to first chunk the plate and then to pick individual animals after they have crawled out of the chunk and into the bacterial lawn. If the animals are in the dauer stage (see Genetic mapping and manipulation-Introduction and basics) you may need to wait a day before picking; the worms will develop out of the dauer stage, and the phenotypes can be scored. When transferring heterozygous stocks that are not well balanced it is best to transfer just one worm to each new plate. This allows you to score the progeny of the transferred worm and confirm that you did indeed transfer a heterozygote. To maintain mating stocks (stocks with both males and hermaphrodites) transfer 6-8 adult males and 3-4 young adult hermaphrodites per plate. You may allow homozygous stocks to starve for several weeks between transfers. Stocks grown at $25^{\circ} \mathrm{C}$ will starve several days sooner than identical stocks grown at $16^{\circ} \mathrm{C}$. It is possible to keep stock plates at $11^{\circ} \mathrm{C}$ or $16^{\circ} \mathrm{C}$ for several months between transfers. To prevent a plate from drying out, wrap it with a strip of Parafilm. Alternatively, you may want to transfer your stocks every 1-2 days so that you have a source of animals at every stage of development.

\subsection{General stock maintenance tips}

C. elegans stocks can best be maintained between $16^{\circ} \mathrm{C}$ and $25^{\circ} \mathrm{C}$, most typically at $20^{\circ} \mathrm{C}$. C. elegans grows 2.1 times faster at $25^{\circ} \mathrm{C}$ than at $16^{\circ} \mathrm{C}$, and 1.3 times faster at $20^{\circ} \mathrm{C}$ than at $16^{\circ} \mathrm{C}$ (Maniatis et al., 1982). This variation in growth periods can be useful when planning experiments.

Take note of specific growth requirements (e.g., temperature sensitivity or dauer defective) and maintain your stocks accordingly. Such information is included on the Strain Information Sheet that is sent with each strain shipped from the CGC.

Stock plates do not need to be stored in a covered container. They will eventually dry out, but this won't be a problem if you transfer animals before the plates reach that condition. If you do store your plates in a covered container be sure to watch for signs of high humidity. Although rare, it is possible for worms to crawl (or be carried in a drop of water) from one plate to another in such conditions, resulting in the mixing of strains.

When pouring or seeding plates, or when transferring worms, it is best to keep the amount of time the cover is removed at a minimum. This cuts down on contamination. If you have mold on a plate, carefully wrap the plate with a strip of Parafilm and dispose of it. Do not take the cover off of a contaminated plate or you risk contaminating other plates. If you must transfer from a contaminated plate, see the section below on Cleaning contaminated stocks.

Label the bottom of the petri plate with the strain name and date. Don't label only the cover, as it may accidentally be separated from the bottom. Discarded plates should be placed in a plastic bag and autoclaved.

\section{Growth of $C$. elegans in liquid medium}

Large quantities of $C$. elegans can be grown in liquid medium. Liquid cultures of $C$. elegans are usually grown on S Medium using concentrated E. coli OP50 as a food source (Lewis and Fleming, 1995). It is often best to grow just one generation of worms in liquid before the worms are harvested. When growing worms for more than one generation, overcrowding can often lead to dauer formation despite the presence of food.

Overnight cultures of E. coli OP50 grown in LB or other rich broth medium should be used to make a concentrated pellet of bacteria. The concentrated pellet can be stored at $4^{\circ} \mathrm{C}$ for several weeks or in a $-70^{\circ} \mathrm{C}$ freezer indefinitely. It is a good idea to have a large amount of concentrated E. coli OP50 available so that food can be 
added as needed. The amount of food needed will depend on the starting inoculum of worms and the length of time the worms are grown. As a point of reference, you can grow a $250 \mathrm{ml}$ batch of liquid culture by inoculating with worms recovered from 4 large $(100 \mathrm{~mm})$ petri plates and growing for 4-5 days. Initially, packed E. coli OP50 from a 2 litre culture is provided. The worm culture is monitored, and additional E. coli OP50 is added as needed.

Protocol 2. Preparation of liquid culture of C. elegans

\section{Reagents}

- $\quad \mathrm{S}$ Basal $\left[5.85 \mathrm{~g} \mathrm{NaCl}, 1 \mathrm{~g} \mathrm{~K}_{2} \mathrm{HPO}_{4}, 6 \mathrm{~g} \mathrm{KH}_{2} \mathrm{PO}_{4}, 1 \mathrm{ml}\right.$ cholesterol $\left(5 \mathrm{mg} / \mathrm{ml}\right.$ in ethanol), $\mathrm{H}_{2} \mathrm{O}$ to 1 litre. Sterilize by autoclaving.]

- 1 M Potassium citrate $\mathrm{pH} 6.0$ [20 g citric acid monohydrate, $293.5 \mathrm{~g}$ tri-potassium citrate monohydrate, $\mathrm{H}_{2} \mathrm{O}$ to 1 litre. Sterilize by autoclaving.]

- $\quad$ Trace metals solution [1.86 g disodium EDTA, $0.69 \mathrm{~g} \mathrm{FeSO}_{4} \bullet 7 \mathrm{H}_{2} \mathrm{O}, 0.2 \mathrm{~g} \mathrm{MnCl} \bullet 4 \mathrm{H}_{2} \mathrm{O}, 0.29 \mathrm{~g} \mathrm{ZnSO}_{4}$ $\bullet 7 \mathrm{H}_{2} \mathrm{O}, 0.025 \mathrm{~g} \mathrm{CuSO}_{4} \bullet 5 \mathrm{H}_{2} \mathrm{O}, \mathrm{H}_{2} \mathrm{O}$ to 1 litre. Sterilize by autoclaving. Store in the dark. $]^{2}$

- $\quad 1 \mathrm{M} \mathrm{CaCl}_{2}\left[55.5 \mathrm{~g} \mathrm{CaCl}_{2}\right.$ in 1 litre $\mathrm{H}_{2} \mathrm{O}$. Sterilize by autoclaving. $]$

- $\quad \mathrm{S}$ Medium [1 litre S Basal, $10 \mathrm{ml} 1 \mathrm{M}$ potassium citrate $\mathrm{pH}$ 6, $10 \mathrm{ml}$ trace metals solution, $3 \mathrm{ml} 1 \mathrm{M} \mathrm{CaCl}_{2}$, $3 \mathrm{ml} 1 \mathrm{M} \mathrm{MgSO}_{4}$. Add components using sterile technique; do not autoclave.]

- 4 large plates of C. elegans, just cleared of bacteria

- $\quad$ concentrated E. coli OP50

\section{Methods}

1. Add $250 \mathrm{ml} \mathrm{S}$ Medium to a sterilized 1-2 litre flask.

2. Inoculate the $\mathrm{S}$ Medium with a concentrated E. coli OP50 pellet made from 2-3 litres of an overnight culture.

3. Wash each of 4 large plates of $C$. elegans (just cleared of bacteria) with $5 \mathrm{ml} \mathrm{S}$ Medium and add to the 250 $\mathrm{ml}$ flask.

4. Put the flask on a shaker at $20^{\circ} \mathrm{C}$. Use fairly vigorous shaking so that the culture is well oxygenated.

5. Cultures should be monitored by checking a drop of the culture under the microscope. If the food supply is depleted (the solution is no longer visibly cloudy) add more concentrated E. coli OP50 suspended in S Medium. When there are many adult animals in each drop, the culture is ready to be harvested. This is usually on the $4^{\text {th }}$ or $5^{\text {th }}$ day.

6. Put the flask on ice for 15 minutes to allow the worms to settle.

7. Aspirate most of the liquid from the flask.

8. Transfer the remaining liquid to a $50 \mathrm{ml}$ sterile conical centrifuge tube and spin for at least $2 \mathrm{~min}$ at $1150 \times$ $\mathrm{g}$ to pellet the worms. Young larvae may take longer than 2 min to pellet.

9. Aspirate the remaining liquid.

The worms harvested from growth in liquid culture are usually longer and thinner than those grown on petri plates, and tend to hold their eggs. The number of adults, larvae and eggs obtained depends on the strain of worms used, the amount of bacteria provided and the length of time the culture is grown. Freshly cleared cultures will yield worms equal to half the weight of the bacteria used (Lewis and Fleming, 1995). 


\section{Cleaning contaminated $C$. elegans stocks}

Occasionally, $C$. elegans stocks may become contaminated with other bacteria, yeast or mold. It is easy to rid your worm stocks of contaminants. Most contaminants will not hurt the worms. (In fact, the worms like to climb some fungal hyphae and sway back and forth!) But it is much easier to score phenotypes and do transfers when your stocks are clean. Mold can be removed by chunking (Protocol 3) and serially transferring, allowing the worms to crawl away from the contaminant. Bacterial contaminants and yeast are easily removed by treating with a hypochlorite solution, which will kill the contaminant and all worms not protected by the egg shell. This can be done using an entire plate that is contaminated (Protocol 4), or it can be done quickly using a single hermaphrodite (Protocol 5).

Protocol 3. Removing mold contaminants from C. elegans stock plates

\section{Methods}

1. Sterilize a scalpel or spatula in a flame and remove a chunk of the agar from the contaminated plate. Remove the cover of the contaminated plate only as long as necessary.

2. Place the chunk of agar at the edge of a seeded clean plate. Allow the worms to crawl out of the chunk and across the E. coli OP50 lawn to the opposite side of the plate.

3. Once the worms have reached the other side of the plate, pick individual animals with a worm picker (or take another chunk using a flamed scalpel or spatula) and place it on the edge of another clean plate.

4. Repeat step 3.

Protocol 4. Egg prep: Removing bacterial or yeast contaminants from C. elegans stock plates

Reagents

- $\quad 5 \mathrm{~N} \mathrm{NaOH}$

- $\quad$ Household bleach (5\% solution of sodium hypochlorite)

Methods

1. Use contaminated $C$. elegans stock plates that have many gravid hermaphrodites. ${ }^{\dagger}$ Wash the plates with sterile $\mathrm{H}_{2} \mathrm{O}$. Pipet the $\mathrm{H}_{2} \mathrm{O}$ across the plate several times to loosen worms and eggs that are stuck in the bacteria.

2. Collect the liquid in a sterile $5 \mathrm{ml}$ conical centrifuge tube with cap. Add $\mathrm{H}_{2} \mathrm{O}$ to total $3.5 \mathrm{ml}$.

3. Mix $0.5 \mathrm{ml} 5 \mathrm{~N} \mathrm{NaOH}$ with $1 \mathrm{ml}$ bleach. Make this solution fresh just before use! Add to the centrifuge tube with the worms.

4. Shake well or vortex the tube for a few seconds. Repeat shaking/vortexing every 2 minutes for a total of 10 minutes.

5. Spin the tube in a table top centrifuge for 30 seconds at $1300 \mathrm{x} g$ to pellet released eggs.

6. Aspirate to $0.1 \mathrm{ml}$.

7. Add sterile $\mathrm{H}_{2} \mathrm{O}$ to $5 \mathrm{ml}$. Shake well or vortex for a few seconds.

8. Repeat steps 5 and 6.

\footnotetext{
${ }^{\dagger}$ It is embryos inside egg shells that will survive this procedure.
} 
9. Use a Pasteur pipet to transfer the eggs in the remaining $0.1 \mathrm{ml}$ of liquid to the edge of a clean NGM plate seeded with an E. coli OP50 lawn.

10. The next day the eggs will have hatched and the larvae will have crawled into the E. coli OP50 lawn. Transfer the hatched larvae to a clean NGM plate seeded with a lawn.

Protocol 5. Egg prep in a drop: Small scale method for removing bacterial or yeast contaminants

Reagents

- $\quad 1 \mathrm{~N} \mathrm{NaOH}$

- Household bleach (5\% solution of sodium hypochlorite)

Methods

1. Make a 1:1 mixture of $1 \mathrm{~N} \mathrm{NaOH}$ :bleach. Put a drop of this solution on the edge of a clean NGM plate seeded with an E. coli OP50 lawn.

2. Put several gravid hermaphrodites in the drop. The solution will kill the contaminants and hermaphrodites but will soak into the plate before the embryos hatch.

3. The next day the larvae will have crawled into the E. coli OP50 lawn. Transfer them to a clean NGM plate seeded with an E. coli OP50 lawn.

Protocol 6. Obtaining synchronous cultures of C. elegans

Reagents

- $\quad \mathrm{M} 9$ Buffer $\left[3 \mathrm{~g} \mathrm{KH}_{2} \mathrm{PO}_{4}, 6 \mathrm{~g} \mathrm{Na}_{2} \mathrm{HPO}_{4}, 5 \mathrm{~g} \mathrm{NaCl}, 1 \mathrm{ml} 1 \mathrm{M} \mathrm{MgSO}_{4}, \mathrm{H}_{2} \mathrm{O}\right.$ to 1 litre. Sterilize by autoclaving.]

- $\quad$ axenized C. elegans eggs from 4-8 $100 \mathrm{~mm}$ plates (see Protocol 4)

- $\quad$ concentrated E. coli OP50 (see Section 5)

Methods

1. Aseptically transfer the axenized eggs to $250 \mathrm{ml} \mathrm{M9}$ Buffer in a 1-2 litre flask and allow to incubate overnight at $20^{\circ} \mathrm{C}$ using fairly vigorous shaking to obtained starved L1 animals.

2. Put the flask on ice for 15 minutes to allow the worms to settle

3. Aspirate most of the liquid from the flask (to remove any dauer pheromone accumulated during starvation)

4. Transfer the remaining liquid to a $50 \mathrm{ml}$ sterile conical centrifuge tube and spin for at least $2 \mathrm{~min}$ at $1150 \mathrm{x}$ $\mathrm{g}$ to pellet the worms.

5. Aspirate the remaining liquid.

6. Transfer the worms to $250 \mathrm{ml}$ of S Basal inoculated with concentrated $E$. coli OP50 in a 1-2 litre flask.

7. Moniter by checking a drop of the culture under the microscope. Add more food as necessary. At $20^{\circ} \mathrm{C}$ mid-L1 larvae can be harvested after approximately 8 hours, mid-L2 larvae at approximately 18 hours, mid-L3 larvae at approximately 25 hours and mid-L4 larvae at approximately 37 hours (Sulston and Hodgkin, 1988). 
If only a small number of synchronized animals are needed, the eggs can be added to a thin layer of M9 Buffer in a $60 \mathrm{~mm}$ petri plate and allowed to grow overnight. The starved L1 animals can then be placed on seeded NGM petri plates, and synchronous growth will begin with the reintroduction of food. Animals should be monitored using the microscope and harvested at the desired stages.

A population of dauer animals can be obtained by adding dauer-inducing pheromone to the liquid culture (Vanfleteren, 1980). Dauers can also be obtained by allowing a liquid culture to grow for several days after the culture is cleared of bacteria. The dauers can be separated from other stages by treating with $1 \%$ SDS for 30 min in a rotating tube and then washing once with $\mathrm{H}_{2} \mathrm{O}$ and once with $\mathrm{M} 9$ Buffer.

\section{Freezing and recovery of $C$. elegans stocks}

Caenorhabditis elegans can be frozen and stored indefinitely in liquid nitrogen $\left(-196{ }^{\circ} \mathrm{C}\right)$ (Brenner, 1974). The keys to a successful freeze are using animals at the correct stage of development, the addition of glycerol to the freezing media, and a gradual cooling to $-80^{\circ} \mathrm{C}$. Freshly starved young larvae (L1-L2 stage) survive freezing best. Well-fed animals, adults, eggs and dauers do not survive well. It is best to use several plates of worms that have just exhausted the E. coli OP50 lawn and that contain lots of L1-L2 animals. A $15 \%$ final volume of glycerol in the freezing solution is used. A $1^{\circ} \mathrm{C}$ decrease in temperature per minute is desirable during freezing. This can be achieved by placing the worms (in freezer vials) in a styrofoam container at $-80^{\circ} \mathrm{C}$. The styrofoam container can be either a commercial shipping box (with walls at least $3 / 4$ inch thick) or a small styrofoam box with slots for holding vials. After 12 or more hours at $-80^{\circ} \mathrm{C}$, the freezer vials should be transferred to their permanent freezer location for long term storage.

The CGC uses two solutions for freezing C. elegans: a Liquid Freezing Solution (Brenner, 1974) and Soft Agar Freezing Solution (Leon Avery, personal communication). For long term storage of stocks in liquid nitrogen, Liquid Freezing Solution is recommended. When this solution is used, the worms settle to the bottom of the freezer vial, and no viable animals can be easily retrieved without thawing the entire contents of the vial. A Soft Agar Freezing Solution is useful for freezing working stocks of $C$. elegans. The addition of the agar helps keep the worms suspended throughout the solution. A small scoop of the frozen contents can be taken, and the remainder can be left in the vial and returned to the freezer for later use. Vials frozen using Soft Agar Freezing Solution should be stored at $-80^{\circ} \mathrm{C}$. If kept in liquid nitrogen, the contents become too hard, and the vial will need to be warmed before it is possible to remove a scoop of the contents. The warming period reduces the number of times live worms can be recovered from the vial. When stored at $-80^{\circ} \mathrm{C}$ there is no need to allow the vial to be warmed; the contents are soft enough to be used right away. We recover worms 3-4 times from each vial of soft agar stock.

Worms can be frozen in $1.8 \mathrm{ml}$ cryotubes. The CGC uses Nunc Cryotube Vials (\#65234) with internal threads and freezes six vials of each strain it receives. Two vials are frozen using Soft Agar Freezing Solution and are stored at $-80^{\circ} \mathrm{C}$ for use as working stocks. Four vials are frozen using Liquid Freezing Solution; one is thawed as a tester, and the other three are put in at least two different liquid nitrogen tanks. To fill strain requests, the working stocks that are kept at $-80^{\circ} \mathrm{C}$ are used. When the last vial of a stock stored at $-80^{\circ} \mathrm{C}$ is emptied, the worms are once again frozen using Soft Agar Freezing Solution in order to replace these vials. In theory, the stocks kept in liquid nitrogen will never need to be used since the soft agar stocks are continually replaced as they are depleted.

The recovery of $C$. elegans from stocks stored in liquid nitrogen is in the range of $35-45 \%$ of the total number of animals frozen. This number decreases only slightly after many years of storage in liquid nitrogen. The recovery of stocks stored at $-80^{\circ} \mathrm{C}$ for many years $(>10)$ is not as high as liquid nitrogen, but worms can be safely stored this way for many years (CGC, unpublished data). Of course, a power failure can result in the loss of all stocks kept at $-80^{\circ} \mathrm{C}$, so it is very wise to keep at least one copy of all stocks in liquid nitrogen. Some mutants strains (especially certain Dpy mutants) do not survive freezing as well as wild-type animals.

Protocol 7. Freezing C. elegans using Liquid Freezing Solution

Equipment and Reagents

- $\quad$ S Buffer $\left[129 \mathrm{ml} 0.05 \mathrm{M} \mathrm{K}_{2} \mathrm{HPO}_{4}, 871 \mathrm{ml} 0.05 \mathrm{M} \mathrm{KH}_{2} \mathrm{PO}_{4}, 5.85 \mathrm{~g} \mathrm{NaCl}\right]$

- $\quad$ S Buffer (see above) $+30 \%$ glycerin (v/v) (autoclave) 
- $\quad 1.8 \mathrm{ml}$ cryotube vials

\section{Methods}

1. Use one large, 2-3 medium, or 5-6 small NGM plates that have lots of freshly starved L1-L2 animals. Wash the plates with $0.6 \mathrm{ml} \mathrm{S}$ Buffer for each vial you will freeze. Collect liquid in a sterile test tube.

2. Add an equal volume of S Buffer $+30 \%$ glycerin. Mix well.

3. Aliquot $1.0 \mathrm{ml}$ of mixture into $1.8 \mathrm{ml}$ cryovials labelled with strain name and date.

4. Pack the cryovials in a small styrofoam box with slots for holding microtubes or use a commercial styrofoam shipping box.

5. Place the box in $\mathrm{a}-80^{\circ} \mathrm{C}$ freezer overnight (or for at least 12 hours).

6. The next day transfer the vials to their permanent freezer locations. Thaw one vial as a tester to check how well the worms survived the freezing (See Protocol 9).

Protocol 8. Freezing C. elegans using Soft Agar Freezing Solution

\section{Equipment and Reagents}

- $\quad$ S Buffer (see Protocol 6)

- Soft Agar Freezing Solution [0.58 g NaCl, $0.68 \mathrm{~g} \mathrm{KH}_{2} \mathrm{PO}_{4}, 30 \mathrm{~g}$ glycerol, $0.56 \mathrm{ml} 1 \mathrm{M} \mathrm{NaOH}, 0.4 \mathrm{~g}$ agar, $\mathrm{H}_{2} \mathrm{O}$ to $100 \mathrm{ml}$ (autoclave)]

- $\quad 1.8 \mathrm{ml}$ cryotube vials

Methods

1. Melt Soft Agar Freezing Solution in autoclave or microwave and place in $50^{\circ} \mathrm{C}$ water bath for at least 15 minutes.

2. Use one large, 2-3 medium, or 5-6 small NGM plates that have lots of freshly starved L1-L2 animals. Wash the plates with $0.6 \mathrm{ml} \mathrm{S}$ Buffer for each vial you will freeze. Collect liquid in a covered sterile test tube and place in ice for 15 minutes.

3. Add an equal volume of Soft Agar Freezing Solution to the test tube. Mix well.

4. Aliquot $1 \mathrm{ml}$ of mixture into $1.8 \mathrm{ml}$ cryovials labelled with strain name and date.

5. Pack the cryovials in a small styrofoam box with slots for holding microtubes or use a commercial styrofoam shipping box.

6. Place the box in a $-80^{\circ} \mathrm{C}$ freezer overnight (or for at least 12 hours).

7. The next day transfer the vials to their permanent freezer locations. Take a scoop of frozen mixture from one vial as a tester to check how well the worms survived the freezing (see Protocol 10).

Protocol 9. Thawing C. elegans frozen using Liquid Freezing Solution

Methods

1. Remove a vial from freezer and allow it to thaw at room temperature until all ice has turned to liquid. 
2. Pour the contents onto one large NGM plate with E. coli OP50 lawn. You should see worms wiggling after just a few minutes.

3. After 2-3 days, transfer 10-15 animals individually to separate plates. Allow the animals to reproduce for one generation and score the progeny for correct phenotypes.

Protocol 10. Thawing C. elegans frozen using Soft Agar Freezing Solution

Methods

1. Remove a vial from $-80^{\circ} \mathrm{C}$ freezer and transfer to a small styrofoam box with slots for microtubes.

2. Work quickly so that the solution in the vial does not thaw. Flame a small scoop or spatula and use it to remove $1 / 4-1 / 3 \mathrm{ml}$ of the frozen solution. Place solution on a NGM plate with E. coli OP50 lawn.

3. Return vial to $-80^{\circ} \mathrm{C}$ freezer as quickly as possible.

4. You should see worms wiggling after just a few minutes. After 2-3 days, transfer 10-15 animals individually to separate plates. Allow the animals to reproduce for one generation and score the progeny for correct phenotypes

\section{Acknowledgements}

The author wishes to thank Robert Herman, Ann Rougvie and Todd Starich for their comments.

\section{References}

Avery, L. (1993). Genetics 133, 897. Abstract

Brenner, S. (1974). Genetics 77, 71. Abstract

Byerly, L., Cassada, R.C., and Russell, R.L. (1976). Dev. Biol. 51, 23. Abstract Article

Caldicott, I.M., Larsen, P.L., and Riddle, D.L. (1994). In: Cell biology: a laboratory handbook. (San Diego: Academic Press), pp. 389.

Hartman, P.S. and Herman, R.K. (1982). Genetics 102, 159. Abstract

Hirsh, D., Oppenheim, D., and Klass, M. (1976). Dev. Biol. 49, 200. Abstract Article

Lewis, J.A. and Fleming, J.T. (1995). In: Methods in cell biology, Vol. 48, H.F. Epstein and D.C. Shakes, eds. (San Diego: Academic Press), p. 3.

Maniatis, T., Fritsch, E.F., and Sambrook, J. (1982). Molecular cloning: a laboratory manual. (New York: Cold Spring Harbor Laboratory Press), p. 68.

Rogalski, T.M. and Riddle, D.L. (1988). Genetics 118, 61. Abstract

Sulston, J. and Hodgkin, J. (1988). In: The Nematode Caenorhabditis elegans, W.B. Wood, ed. (New York: Cold Spring Harbor Laboratory Press), p. 587.

Vanfleteren, J.R. (1980). In: Nematodes as biological models, Vol. 2, B.M. Zuckerman, ed. (New York: Academic Press), p. 47. 